\title{
Knockdown of Arf6 increases drug sensitivity and inhibits proliferation, migration and invasion in gastric cancer SGC-7901 cells
}

\author{
JUNLAN QIU $^{1}$, LIANG TAO ${ }^{1}$, QIANG WEI ${ }^{2}$ and PINGYANG ZHANG ${ }^{3}$ \\ ${ }^{1}$ Department of General Surgery, Nanjing Drum Tower Hospital Clinical College, Nanjing Medical University, Nanjing, \\ Jiangsu 210008; ${ }^{2}$ Nanjing Emergency Medical Center, Nanjing, Jiangsu 210003; ${ }^{3}$ Department of Cardiovascular Ultrasound, \\ Nanjing First Hospital Affiliated to Nanjing Medical University, Nanjing, Jiangsu 210006, P.R. China
}

Received February 11, 2017; Accepted November 2, 2017

DOI: $10.3892 / \mathrm{ol} .2017 .7558$

\begin{abstract}
ADP-ribosylation factor 6 (Arf6), a member of the ADP-ribosylation factor family, is overexpressed in different types of cancer cell and promotes invasion, metastasis and drug resistance. However, the potential functions of Arf6 in gastric cancer (GC), and the molecular mechanism underlying these functions, remain to be fully elucidated. In the present study, the results demonstrated that in vitro knockdown of Arf6 decreased proliferation, colony formation, migration and invasion in SGC-7901 cells. Arf6 knockdown also markedly decreased the activity of the extracellular signal-regulated kinase 1/2 (ERK1/2) signaling pathway. Furthermore, knockdown of Arf6 was associated with elevated chemosensitivity of SGC-7901 cells to 5-fluorouracil through inactivation of the ERK1/2 signaling pathway. Taken together, these results suggest that Arf6 is involved in regulating proliferation, migration, invasion and drug resistance in GC, and may be a potential therapeutic target for the treatment of GC.
\end{abstract}

\section{Introduction}

In 2013, gastric cancer (GC) had become the third most common cause of cancer-associated mortality globally (1). It is estimated that 951,600 new cases and 723,100 GC-associated mortalities were recorded worldwide in $2012(2,3)$. Multistep processes and molecular markers have been confirmed to be involved in the tumorigenesis and invasiveness of GC (4). A combination of surgery and chemotherapy has increased the survival time of patients with GC $(5,6)$. However, a significant

Correspondence to: Professor Pingyang Zhang, Department of Cardiovascular Ultrasound, Nanjing First Hospital Affiliated to Nanjing Medical University, 68 Changle Road, Nanjing, Jiangsu 210006, P.R. China

E-mail: pingyangz@163.com

Key words: ADP-ribosylation factor 6, drug resistance, gastric cancer, fluorouracil number of patients still suffer from relapse due to the resistance of tumor cells to chemotherapeutic agents (5). Therefore, understanding the molecular pathways underlying GC carcinogenesis and progression will assist in improving diagnosis, therapy, and prevention of this disease.

ADP-ribosylation factor 6 (Arf6), a member of the Arf family, has emerged as a critical regulator of membrane traffic, cell polarity and cytoskeletal organization $(6,7)$. There is increasing evidence to confirm that Arf6 is associated with cancer development (8-10). Arf6 is overexpressed in various types of human cancer, including breast cancer, lung adenocarcinoma, clear cell renal cell carcinoma and head and neck squamous cell carcinoma (11-13). In glioblastoma cells, early studies have demonstrated that Arf6 is required for epidermal growth factor (EGF)-induced cell proliferation (14). Arf6 has also been implicated in other cellular processes associated with tumorigenesis, including the epithelial-mesenchymal transition (EMT), migration and invasion $(12,15,16)$. Furthermore, activation of Arf6 is associated with drug resistance in the breast cancer cell lines MDA-MB-231, MDA-MB-453 and Hs578T (17-19). Arf6 has also been demonstrated to mediate EGF-induced EMT in GC cells (20). The EMT phenotype in cancers is associated with migration, invasion and drug resistance $(21,22)$. However, the involvement of Arf6 in the growth, migration, invasion and drug resistance of GC cells remains to be fully elucidated.

The present study was designed to explore the function of Arf6 in the proliferation, migration, invasion and drug resistance in GC cells. Our results demonstrate that Arf6 contributes to the proliferation, migration and invasion of GC cells. Knockdown of Arf6 was demonstrated to increase the sensitivity of GC cells to 5 -fluorouracil (5-FU) via inhibition of the extracellular signal-regulated kinase 1/2 (ERK1/2) signaling pathway. Therefore, Arf6 may be a potential target for GC therapy.

\section{Materials and methods}

Cell culture. The human GC cell line SGC-7901 was obtained from the Type Culture Collection of the Institute of Chinese Academy of Sciences (Shanghai, China). Cells were cultured in 
Dulbecco's modified Eagle's medium (DMEM; high glucose) supplemented with $10 \%$ (v/v) fetal bovine serum (FBS) (both from Hyclone; GE Healthcare Life Sciences, Logan, UT, USA) and antibiotics $(100 \mathrm{U} / \mathrm{ml}$ streptomycin and $100 \mu \mathrm{g} / \mathrm{ml}$ penicillin; Invitrogen; Thermo Fisher Scientific, Inc., Waltham, MA, USA) in a humidified incubator at $37^{\circ} \mathrm{C}$ with $5 \% \mathrm{CO}_{2}$.

Small interfering RNA (siRNA) transfection. siRNA sequences were designed and synthesized by Shanghai GenePharma Co., Ltd. (Shanghai, China) according to the Arf6 gene sequence [GenBank (23); accession no. NM 001663.3]. The sequences for Arf6 were as follows: siArf6-1, 5'-GUGGCAAAUAAUGAG UAAUTT-3'; siArf6-2, 5'-GCGACCACUAUGAUAAUAU TT-3'; and siArf6-3, 5'-GACGCCAUAAUCCUCAUCUTT-3'. The control siRNA (siCtr) sequence was 5'-UUCUCCGAAC GUGUCACGUTT-3'. SGC-7901 cells were seeded onto 6-well plates at a density of $10^{5}$ cells/well and were incubated for $24 \mathrm{~h}$. Cells were then transfected with control siRNA or Arf6 siRNA with Lipofectamine 2000 (Thermo Fisher Scientific, Inc.), according to the manufacturer's instructions. The total siRNA concentration in each well was 100 pmol and untransfected controls were used to demonstrate that there was no significant difference between untransfected controls and cells transfected with control siRNA. Arf6 silencing was confirmed by assessing them mRNA and protein expression levels in the SGC-7901 cells $48 \mathrm{~h}$ after transfection, as follows.

Reverse transcription-polymerase chain reaction (RT-PCR). Total RNA was extracted from cells using TRIzol reagent (Invitrogen; Thermo Fisher Scientific, Inc.), according to the manufacturer's instructions. cDNA was synthesized using an RNA to cDNA EcoDry ${ }^{\mathrm{TM}}$ Premix (Oligo dT) kit (Takara Biotechnology Co., Ltd., Dalian, China) according to the manufacturer's instructions. The following gene-specific primers were used in the present study: Arf6 forward, 5'-CAA GGTCTCATCTTCGTAGTG-3' and reverse, 5'-CATGTG AGCCCCTCATAGAG-3'; GAPDH forward, 5'-TGAACG GGAAGCTCACTGG-3' and reverse, 5'-TCCACCACCCTG TTGCTGTA-3'. PCR analysis was performed using Takara Ex Taq ${ }^{\circledR}$ DNA polymerase (Takara Biotechnology Co., Ltd.) under the following thermocycling conditions: $95^{\circ} \mathrm{C}$ for $3 \mathrm{~min}$, followed by 28 cycles of $95^{\circ} \mathrm{C}$ for $30 \mathrm{sec}, 57^{\circ} \mathrm{C}$ (Arf6) or $55^{\circ} \mathrm{C}$ (GAPDH) for $30 \mathrm{sec}$, and $72^{\circ} \mathrm{C}$ for $1 \mathrm{~min}$, with a final extension at $72^{\circ} \mathrm{C}$ for $6 \mathrm{~min}$. The PCR products were electrophoresed on $1 \%$ agarose gels and the bands were visualized by UV fluorescence following staining with ethidium bromide $(2.5 \mathrm{~g} / \mathrm{ml})$ for $15 \mathrm{~min}$. Data were analyzed by densitometry using Tanon Gel Image System software, version 4.0 (Tanon Science and Technology Co., Ltd., Shanghai, China).

Western blot analysis. Cells were lysed for $5 \mathrm{~min}$ on ice in radioimmunoprecipitation assay buffer (cat. no. 89900; Thermo Fisher Scientific, Inc.) containing 1\% phenylmethanesulfonyl fluoride (Beyotime Institute of Biotechnology, Haimen, China). Lysate was then sonicated and centrifuged at $12,000 \mathrm{x}$ g for $10 \mathrm{~min}$ at $4^{\circ} \mathrm{C}$. The protein concentration was quantified using a bicinchoninic acid assay (Beyotime Institute of Biotechnology). Proteins from each sample $(20 \mu \mathrm{g})$ were subjected to $10 \%$ SDS-polyacrylamide gel electrophoresis and transferred to polyvinylidene difluoride membranes. The membranes were then blocked with 5\% skim milk at room temperature for $1 \mathrm{~h}$ and incubated overnight with mouse anti-Arf6 (1:500 dilution, cat. no. sc-7971; Santa Cruz Biotechnology, Inc., Dallas, TX, USA), rabbit anti-ERK1/2 (1:1,000 dilution, cat. no. 4695; Cell Signaling Technology, Inc., Danvers, MA, USA), rabbit anti-phosphorylated ERK1/2 (pERK1/2) (1:1,000 dilution, cat. no. 4377; Cell Signaling Technology, Inc.), and mouse anti-GAPDH (1:2,000 dilution, cat. no. sc-47724; Santa Cruz Biotechnology, Inc.) overnight at $4^{\circ} \mathrm{C}$. Following washing with TBST 3 times, the membranes were incubated with goat anti-rabbit IgG (1:3,000 dilution, cat. no. sc-2004; Santa Cruz Biotechnology, Inc.) or goat anti-mouse IgG (1:3,000 dilution, cat. no. sc-2005; Santa Cruz Biotechnology, Inc.) horseradish peroxidase-conjugated secondary antibodies for $1 \mathrm{~h}$ at $37^{\circ} \mathrm{C}$, and visualized using enhanced chemiluminescence detection reagents (Thermo Fisher Scientific, Inc.) and were exposed to chemiluminescent film (Thermo Fisher Scientific, Inc.). Data were analyzed using ImageJ software (version 1.6; National Institutes of Health, Bethesda, MD, USA) and were normalized to GAPDH expression.

Cell proliferation assay. Cell proliferation was measured using Cell Counting Kit-8 (CCK-8) according to the manufacturer's instructions (Sigma-Aldrich; Merck KGaA, Darmstadt, Germany). Briefly, control and transfected cells were seeded at a density of $3 \times 10^{3}$ cells/well in 96-well plates and treated with $0,1,10,25,50,100 \mu \mathrm{g} / \mathrm{ml} 5-\mathrm{FU}$ for $48 \mathrm{~h}$ at $37^{\circ} \mathrm{C}$ or $10 \mu \mathrm{M}$ ERK1/2 inhibitor U0126 (both from Sigma-Aldrich; Merck KGaA) for $12 \mathrm{~h}$ prior to being treated with $20 \mu \mathrm{g} / \mathrm{ml}$ 5-FU for $48 \mathrm{~h}$ at $37^{\circ} \mathrm{C}$. CCK-8 $(10 \mu \mathrm{l})$ was added to each well and incubated for an additional $4 \mathrm{~h}$ at $37^{\circ} \mathrm{C}$. Optical density (OD) was measured using a microplate reader (Omega Bio-Tek, Inc., Norcross, GA, USA) at $450 \mathrm{~nm}$. Each time-point was repeated in three wells, and the experiment was independently performed three times.

Colony formation assay. Control and transfected cells were seeded at a density of $5 \times 10^{2}$ cells/well in 6-well plates, and cultured in DMEM in an environment with $5 \% \mathrm{CO}_{2}$ at $37^{\circ} \mathrm{C}$ for 14 days to allow colonies to form. The plates were stained with $0.5 \%(\mathrm{w} / \mathrm{v})$ crystal violet in $70 \%$ ethanol for $20 \mathrm{~min}$ at room temperature, and colonies were counted under a light microscope (TS100; Nikon Corporation, Tokyo, Japan). The experiment was independently performed three times.

In vitro migration and invasion assays. For the Transwell migration assay, untransfected and transfected SGC-7901 cells in the exponential growth phase were trypsinized with $1 \mathrm{X}$ trypsin, washed twice with phosphate-buffered saline, and suspended in DMEM without FBS. Cells $\left(2 \times 10^{4}\right.$ cells/well $)$ were seeded into polycarbonate membrane inserts $(8-\mu \mathrm{m}$ pore size) in 24-well Transwell cell culture dishes. Cells were permitted to attach to the membrane for $30 \mathrm{~min}$. The lower chamber was filled with $600 \mu$ l DMEM with $10 \%$ FBS. Cells were permitted to migrate for $24 \mathrm{~h}$ at $37^{\circ} \mathrm{C}$. Following incubation, stationary cells were removed from the upper surface of the membranes. The cells that had migrated to the lower surface were fixed with $4 \%$ paraformaldehyde at room temperature for $15 \mathrm{~min}$ and were stained with $0.1 \%$ crystal violet for $15 \mathrm{~min}$ at the same temperature. The cells that had migrated 
A
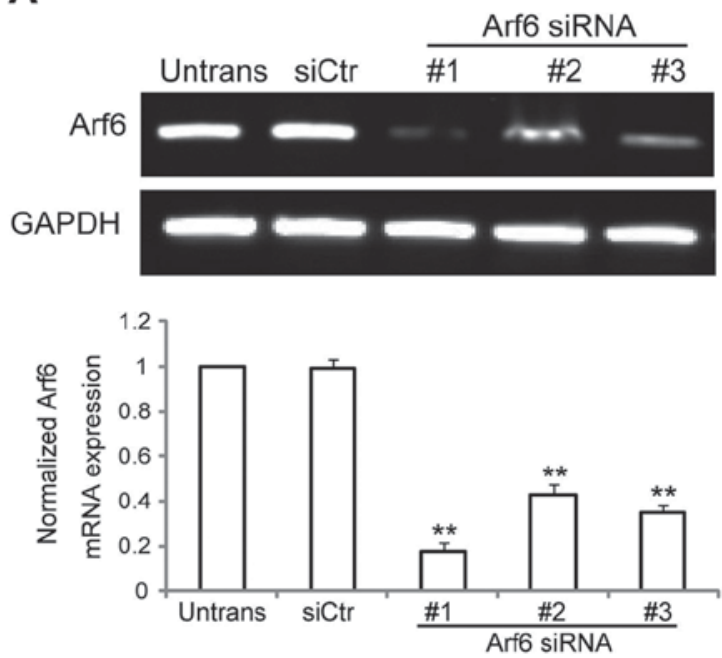

B
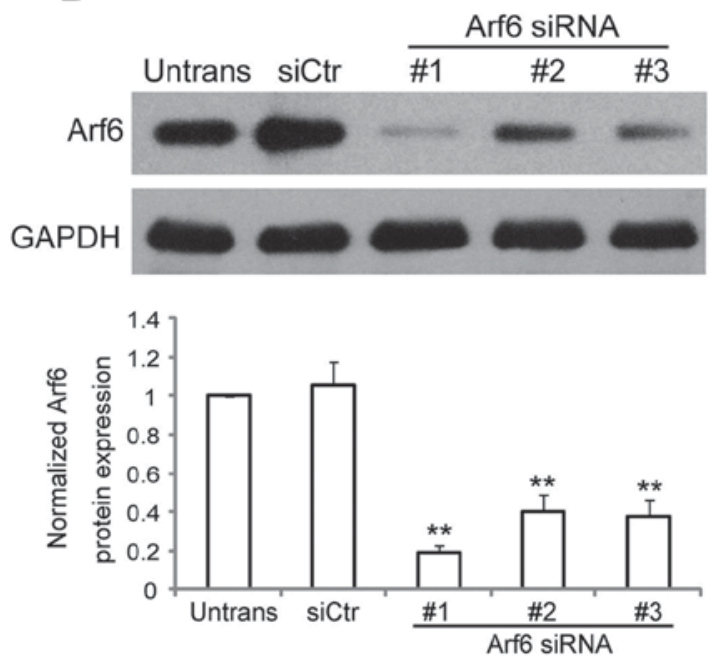

Figure 1. Inhibition of Arf6 expression in SGC-7901 cells by siRNA. (A) Cells were transfected with three siRNA oligonucleotides, and the expression of Arf6 mRNA was analyzed using reverse transcription polymerase chain reaction $48 \mathrm{~h}$ following transfection. (B) The expression of Arf6 protein in SGC-7901 cells was analyzed following transfection with the siRNA oligonucleotides by western blot analysis. GAPDH served as a loading control. ${ }^{* *} \mathrm{P}<0.01$ vs. siCtr. Arf6, ADP-ribosylation factor 6; siRNA, small interfering RNA; siCtr, control siRNA; Untrans, untransfected.

through the membrane were manually counted at x200 magnification from 5 fields/filter using a light microscope (TS100; Nikon Corporation). The invasion assay was performed using Matrigel-coated chambers from the BioCoat Matrigel Invasion Chamber kit (BD Biosciences, Franklin Lakes, NJ, USA) using the same method as aforementioned for the migration assay.

Statistical analysis. Data were statistically analyzed using SPSS 17.0 software (SPSS, Inc., Chicago, IL, USA). Data were presented as the mean \pm SD of three independent experiments. Differences between experimental groups were analyzed using one way analysis of variance (ANOVA). The Student-Newman-Keuls test was used as a post hoc test following ANOVA. $\mathrm{P}<0.05$ was considered to indicate a statistically significant difference.

\section{Results}

Knockdown of Arf6 in SGC-7901 cells by siRNA. To investigate the function of Arf6 in GC cells, our group identified and validated three independent and non-overlapping siRNA sequences to deplete endogenous Arf6 expression in SGC-7901 cells. RT-PCR and western blot analysis were used to evaluate the ability of different Arf6 siRNAs to silence Arf6 expression in vitro. As presented in Fig. 1A and B, Arf6 expression was significantly reduced in cells transfected with specific siRNAs against Arf6. Arf6 mRNA expression was reduced by $\sim 82 \%$ with siArf6-1, $57 \%$ with siArf6-2 and $\sim 65 \%$ with siArf6-3, while protein expression was reduced by $\sim 81 \%$ with siArf6-1, $\sim 60 \%$ with siArf6-2 and $\sim 62 \%$ with siArf6-3, compared with cells transfected with siCtr. Thus, siArf6-1 was selected as the most efficient and specific sequence to silence the expression of Arf6 in the SGC-7901 cells, and was used in all subsequent experiments.

Knockdown of Arf6 inhibits the proliferation of SGC-7901 cells in vitro. The effect of Arf6 knockdown on cell proliferation and tumorigenesis were assessed using CCK-8 and colony formation assays, respectively. As presented in Fig. 2A, Arf6 knockdown resulted in a significant decrease in the proliferation of SGC-7901 cells at 48, 72, and 96 h. In addition, cells transfected with siArf6 formed fewer and smaller colonies as compared with cells transfected with siCtr (Fig. 2B). Taken together, these data indicate that knockdown of Arf6 resulted in a significant inhibitory effect on cell proliferation and colony formation in SGC-7901 cells in vitro.

Knockdown of Arf6 inhibits the migration and invasion of SGC-7901 cells in vitro. The in vitro migration and invasion assays were designed to investigate the function of Arf6 in SGC-7901 cell migratory and invasive processes. For the migration assay, untransfected and transfected cells were seeded on Transwell chambers with uncoated filters. In total, $100 \%$ of the untransfected SGC-7901 cells were able to migrate to the filters in $24 \mathrm{~h}$, while the migratory percentage of siCtr-transfected cells was $98 \%$ and that of siArf6-transfected cells was $38 \%$ (Fig. 3A). For the invasion assay, untransfected and transfected cells were seeded on Transwell chambers with Matrigel-coated filters. After $24 \mathrm{~h}$ of incubation, the invasion of siArf6 cells was significantly reduced (Fig. 3B). Taken together, these results indicated that silencing Arf6 reduces SGC-7901 cell migration and invasion in vitro.

Knockdown of Arf6 decreases activation of the ERK1/2 pathway. According to the results of previous studies, Arf6 regulates the activation of ERK1/2 (24-26). Furthermore, activation of ERK1/2 has been demonstrated to increase cell proliferation, migration and invasion in GC (27-29). Thus, the effect of Arf6 knockdown on the ERK1/2 pathway was investigated in SGC-7901 cells. As presented in Fig. 4A, p-ERK1/2 levels were significantly reduced in the Arf6-knocdown SGC-7901 cells, while total ERK1/2 expression was comparable to that observed in the control cells. This association between Arf6 and p-ERK1/2 expression suggested that Arf6 
A

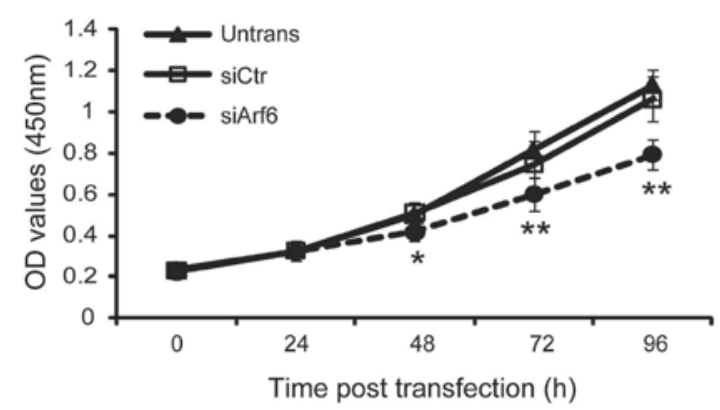

B

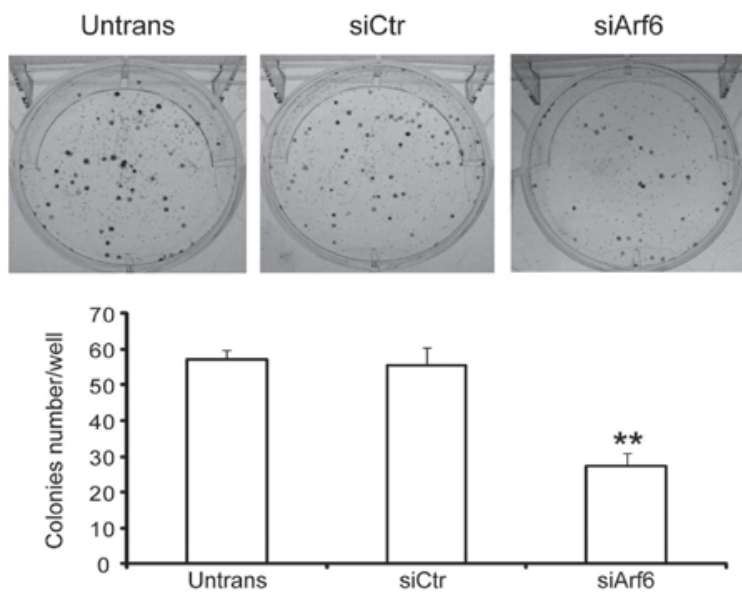

Figure 2. Knockdown of Arf6 inhibits cell proliferation and colony formation of SGC-7901 cells in vitro. (A) Cell proliferation was analyzed using a Cell Counting Kit-8 assay. (B) Untransfected and transfected SGC-7901 cells were cultured for 14 days to allow colonies to form. Positive colonies were counted under a microscope. The accompanying graph summarizes the colony-forming capacities of the cells. ${ }^{*} \mathrm{P}<0.05$ and ${ }^{* *} \mathrm{P}<0.01$ vs. siCtr. Arf6, ADP-ribosylation factor 6; siCtr, control siRNA; Untrans, untransfected; siArf6, siRNA targeting Arf6.

may be involved in the regulation of the ERK1/2 signaling pathway.

Knockdown of Arf6 enhances sensitivity of SGC-7901 cells to 5-FU through modulating the ERK1/2 signaling pathway. Previous studies have reported that Arf6 is involved in drug resistance in a variety of types of cancer cell (17-19). The effect of Arf6 knockdown on the sensitivity of SGC-7901 cells to 5 -FU was further investigated. To determine the sensitivity of cells to 5-FU, siArf6 or siCtr-transfected SGC-7901 cells were exposed to different concentrations of 5-FU, ranging from 0 to $100 \mu \mathrm{g} / \mathrm{ml}$, for $48 \mathrm{~h}$. Cell viability was examined using a CCK- 8 assay. The cell survival rate appeared to show a dose-dependent decrease in response to 5-FU treatment, and knockdown of Arf6 resulted in increased sensitivity to 5-FU treatment in SGC-7901 cells (Fig. 4B). Next, the present study aimed to determine whether the Arf6 knockdown-enhanced 5-FU sensitivity of SGC-7901 cells was due to inactivation of the ERK1/2 signaling pathway. As presented in Fig. 4C, 5-FU suppressed the phosphorylation of ERK1/2 in SGC-7901 cells. However, knockdown of Arf6 resulted in a smaller reduction of p-ERK1/2 expression, while total ERK1/2 expression was
A
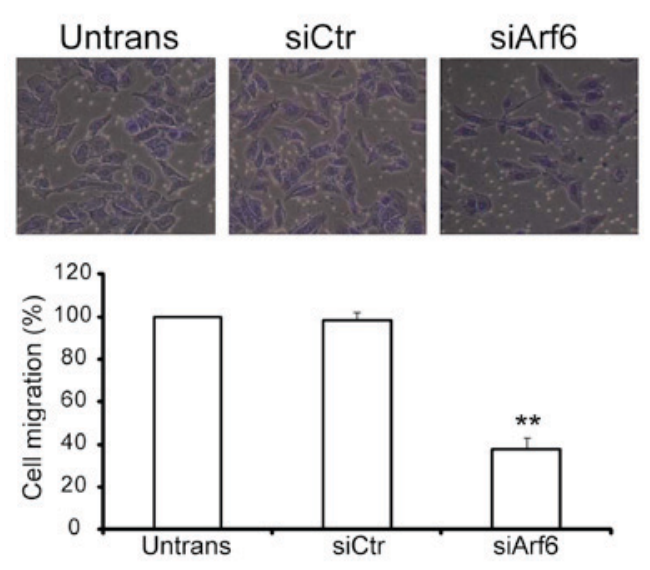

B
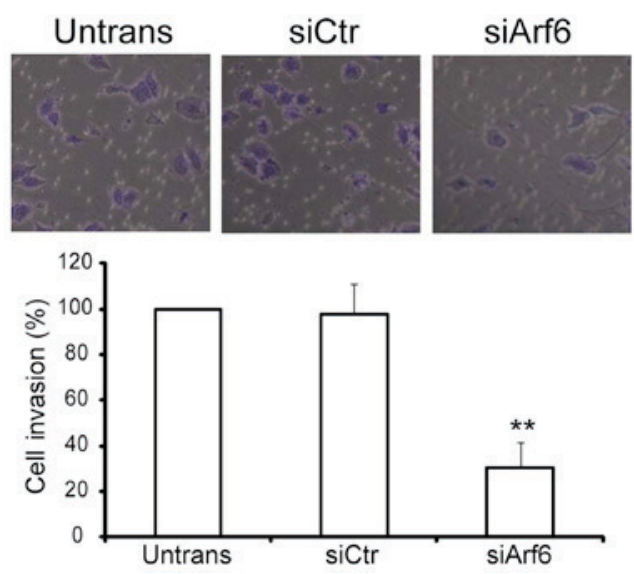

Figure 3. Knockdown of Arf6 inhibits the migration and invasion of SGC-7901 cells in vitro. (A) Results of the migration assay (magnification, $\mathrm{x} 200$ ). (B) Results of the invasion assay (magnification, $\mathrm{x} 200$ ). ${ }^{* *} \mathrm{P}<0.01$ vs. siCtr. Arf6, ADP-ribosylation factor 6; siCtr, control siRNA; Untrans, untransfected; siArf6, siRNA targeting Arf6.

unaffected. The CCK-8 results revealed that U0126 (a specific MEK inhibitor) effectively increased siArf6-mediated 5-FU sensitivity of SGC-7901 cells (Fig. 4D). Collectively, these results indicated that knockdown of Arf6 enhanced the chemosensitivity of SGC-7901 cells to 5-FU by suppressing ERK1/2 activity.

\section{Discussion}

Arf6 is a member of the Arf family which exhibits pleiotropic biological functions (7,30-33). Arf6 has been reported to be upregulated in multiple types of tumor, and has been demonstrated to be involved in a number of biological processes, including cancer cell growth, EMT, cell adhesion, migration, invasion, angiogenesis, malignant transformation and resistance to chemotherapy $(18,24,34-36)$. However, the biological functions of Arf6 in GC remain to be fully elucidated.

To investigate the potential associations between Arf6 expression and the biological features of GC cells, Arf6 expression was knocked down in GC cells using three siRNA 
A

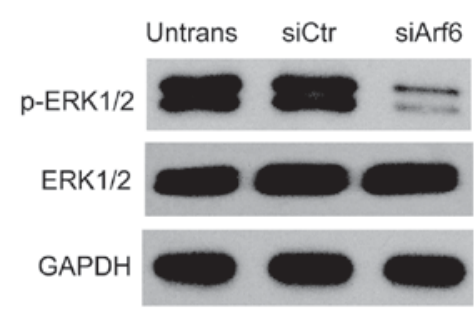

C
B

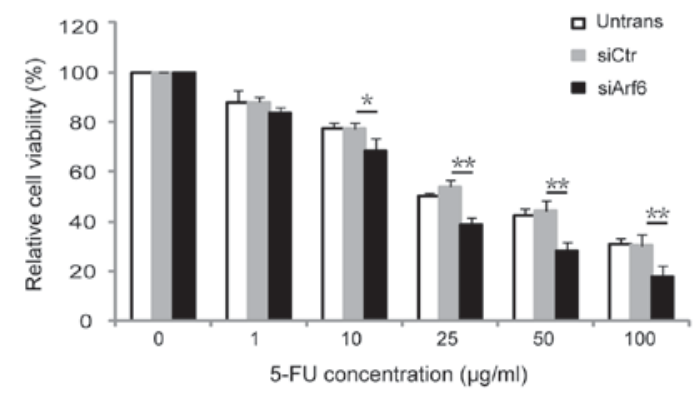

D
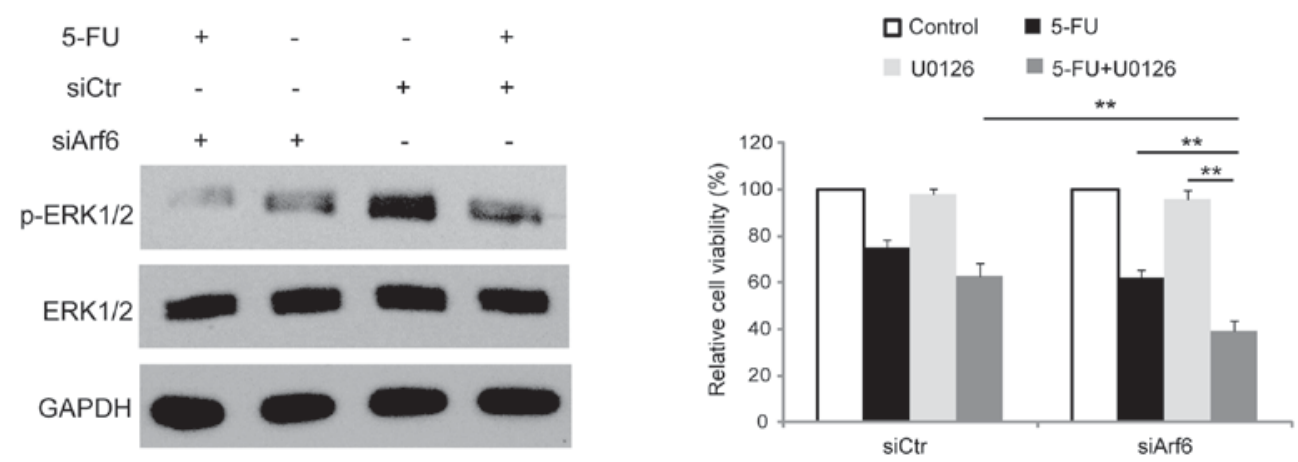

Figure 4. Knockdown of Arf6 increases the sensitivity of SGC-7901 cells to 5-FU through modulation of ERK1/2 signaling. (A) SGC-7901 cells were transfected with siCtr or siArf6, and $48 \mathrm{~h}$ following transfection proteins were extracted and subjected to western blot analysis. (B) Dose-dependent CCK-8 assay was employed to investigate the effect of Arf6-knockdown on the viability of SGC-7901 cells. (C) Western blot analysis of ERK1/2 and p-ERK1/2 in SGC-7901 cells transfected with siCtr or siArf6 and treated with $20 \mu \mathrm{g} / \mathrm{ml} 5$-FU for $48 \mathrm{~h}$. (D) SGC-7901 cells transfected with siCtr or siArf6 were treated with $10 \mu \mathrm{M}$ U0126 for $12 \mathrm{~h}$ prior to being treated with 5-FU, and cell viability was determined using a CCK-8 assay. ${ }^{*}<0.05$ and ${ }^{* *} \mathrm{P}<0.01$ vs. siCtr. Arf6, ADP-ribosylation factor 6; 5-FU, 5-fluorouracil; siCtr, control siRNA; siArf6, siRNA targeting Arf6; CCK-8, Cell Counting Kit-8; ERK1/2, extracellular signal-regulated kinase 1/2; p, phosphorylated; Untrans, untransfected.

sequences, and siRNA targeting of Arf6 in SGC-7901 cells in vitro resulted in efficient, specific inhibition of endogenous Arf6 mRNA and protein. Further experiments demonstrated that knockdown of Arf6 in SGC-7901 cells significantly inhibited the migration and invasion of SGC-7901 cells in vitro. These results indicated that Arf6 expression is associated with pro-metastatic events in SGC-7901 cells. These data are consistent with previous results in other tumor cell lines, including breast cancer cells (37) and lung cancer cells (13). Furthermore, Arf6 has also been implicated in the modulation of cancer cell growth and the tumorigenic phenotype of cancer cells in pancreatic and lung cancer $(10,35)$. The present study also demonstrated that Arf6-knockdown SGC-7901 cells had reduced proliferation and a reduced ability to form colonies. Taken together, these results suggest that Arf6 expression is associated with migration, invasion, proliferation and tumorigenicity in SGC-7901 cells.

Previous studies have demonstrated the presence of an association between Arf6 and ERK1/2 signaling in several cancer cell lines, and this association has been implicated in cancer progression $(20,24,25)$. Furthermore, ERK1/2 signaling has been demonstrated to mediate cell proliferation, migration and invasion in various types of tumor cell, including GC cells (27-29). In the present study, the effect of Arf6 knockdown on ERK1/2 activation was investigated in SGC-7901 cells. Phosphorylation of ERK1/2 was markedly reduced in Arf6 siRNA-transfected cells compared with the control cells, indicating that the migration, invasion, proliferation and tumorigenicity of SGC-7901 cells are regulated via the ERK1/2 pathway. However, the precise mechanisms by which Arf6 knockdown inhibits tumor growth, migration and invasion require further study.

Previous studies have demonstrated that Arf6 confers resistance to multiple chemotherapy agents, including gemcitabine, fluorouracil and temsirolimus (17-19). However, whether Arf6 is involved in chemoresistance in GC cells specifically remains unclear. In the present study, knockdown of Arf6 was revealed to sensitize SGC-7901cells to 5-FU in vitro, suggesting that Arf6 induces 5-FU resistance in GC cells. Inhibition of the ERK1/2 pathway has been reported to increase 5-FU efficacy in multiple cancer cell lines, including GC cell lines. Furthermore, the results of the present study demonstrated that Arf6 knockdown significantly decreased ERK1/2 signaling pathway activity. Thus, whether Arf6 regulates chemosensitivity to 5 -FU by modulating ERK1/2 in SGC-7901 cells was investigated. The results revealed that the specific ERK1/2 inhibitor U0126 effectively increased Arf6 siRNA-mediated 5-FU sensitivity. These results indicated that Arf6 may regulate chemosensitivity to 5-FU through the ERK1/2 signaling pathway in SGC-7901 cells. 
In conclusion, the results of the present study demonstrated that knockdown of Arf6 inhibits SGC-7901 cell proliferation, migration and invasion, and increases the sensitivity of SGC-7901 cells to 5-FU, with the increasing drug sensitivity potentially associated with the inhibition of ERK1/2 signals. Understanding the mechanisms underlying these effects may provide novel strategies for GC treatment. Combining Arf6 gene therapy with traditional chemotherapy may be an effective anti-GC strategy in the future.

\section{References}

1. Piazuelo MB and Correa P: Gastric cáncer: Overview. Colomb Med (Cali) 44: 192-201, 2013.

2. Siegel R, Ma J, Zou Z and Jemal A: Cancer statistics, 2014. CA Cancer J Clin 64: 9-29, 2014.

3. Qi X, Liu Y, Wang W, Cai D, Li W, Hui J, Liu C, Zhao Y and Li G: Management of advanced gastric cancer: An overview of major findings from meta-analysis. Oncotarget 7: 78180-78205, 2016.

4. Akhavan-Niaki H and Samadani AA: Molecular insight in gastric cancer induction: An overview of cancer stemness genes. Cell Biochem Biophys 68: 463-473, 2014.

5. Flotow $\mathrm{H}$ : The use of high-throughput screening in identifying chemotherapeutic agents for gastric cancer. Future Med Chem 6 : 2103-2112, 2014

6. Mukhamedova N, Hoang A, Cui HL, Carmichael I, Fu Y, Bukrinsky $M$ and Sviridov D: Small GTPase ARF6 regulates endocytic pathway leading to degradation of ATP-binding cassette transporter A1. Arterioscler Thromb Vasc Biol 36 2292-2303, 2016.

7. Zhu X, Zhou T, Chen L, Zheng S, Chen S, Zhang D, Li G and Wang Z: Arf6 controls endocytosis and polarity during asexual development of Magnaporthe oryzae. FEMS Microbiol Lett 363: fnw248, 2016.

8. Hashimoto S, Mikami S, Sugino H, Yoshikawa A, Hashimoto A, Onodera Y, Furukawa S, Handa H, Oikawa T, Okada Y, et al: Lysophosphatidic acid activates Arf6 to promote the mesenchymal malignancy of renal cancer. Nat Commun 7: 10656, 2016

9. Hongu T, Yamauchi Y, Funakoshi Y, Katagiri N, Ohbayashi N and Kanaho Y: Pathological functions of the small GTPase Arf6 in cancer progression: Tumor angiogenesis and metastasis. Small GTPases 7: 47-53, 2016.

10. Liang C, Qin Y, Zhang B, Ji S, Shi S, Xu W, Liu J, Xiang J, Liang D, Hu Q, et al: ARF6, induced by mutant Kras, promotes proliferation and Warburg effect in pancreatic cancer. Cancer Lett 388: 303-311, 2016.

11. Hashimoto S, Onodera Y, Hashimoto A, Tanaka M, Hamaguchi M, Yamada A and Sabe H: Requirement for Arf6 in breast cancer invasive activities. Proc Natl Acad Sci USA 101: 6647-6652, 2004.

12. Morishige M, Hashimoto S, Ogawa E, Toda Y, Kotani $\mathrm{H}$ Hirose M, Wei S, Hashimoto A, Yamada A, Yano $\mathrm{H}$, et al: GEP100 links epidermal growth factor receptor signalling to Arf6 activation to induce breast cancer invasion. Nat Cell Biol 10: 85-92, 2008.

13. Oka S, Uramoto H, Shimokawa H, Yamada S and Tanaka F: Epidermal growth factor receptor-GEP100-Arf6 axis affects the prognosis of lung adenocarcinoma. Oncology 86: 263-270, 2014.

14. Li M, Wang J, Ng SS, Chan CY, He ML, Yu F, Lai L, Shi C, Chen Y, Yew DT, et al: Adenosine diphosphate-ribosylation factor 6 is required for epidermal growth factor-induced glioblastoma cell proliferation. Cancer 115: 4959-4972, 2009.

15. Hashimoto A, Hashimoto S, Ando R, Noda K, Ogawa E, Kotani H, Hirose M, Menju T, Morishige M, Manabe T, et al: GEP100-Arf6-AMAP1-cortactin pathway frequently used in cancer invasion is activated by VEGFR2 to promote angiogenesis. PLoS One 6: e23359, 2011.

16. Matsumoto Y, Sakurai H, Kogashiwa Y, Kimura T, Matsumoto Y, Shionome T, Asano M, Saito K and Kohno N: Inhibition of epithelial-mesenchymal transition by cetuximab via the EGFR-GEP100-Arf6-AMAP1 pathway in head and neck cancer. Head Neck 39: 476-485, 2017.

17. Dani N, Barbosa AJ, Del Rio A and Di Girolamo M: ADP-ribosylated proteins as old and new drug targets for anticancer therapy: The example of ARF6. Curr Pharm Des 19: 624-633, 2013
18. Hashimoto A, Hashimoto S, Sugino H, Yoshikawa A, Onodera Y, Handa H, Oikawa T and Sabe H: ZEB1 induces EPB41L5 in the cancer mesenchymal program that drives ARF6-based invasion, metastasis and drug resistance. Oncogenesis 5: e259, 2016.

19. Hashimoto A, Oikawa T, Hashimoto S, Sugino H, Yoshikawa A, Otsuka Y, Handa H, Onodera Y, Nam JM, Oneyama C, et al: P53- and mevalonate pathway-driven malignancies require Arf6 for metastasis and drug resistance. J Cell Biol 213: 81-95, 2016.

20. Zhang Y, Du J, Zheng J, Liu J, Xu R, Shen T, Zhu Y, Chang J, Wang $\mathrm{H}$, Zhang Z, et al: EGF-reduced Wnt5a transcription induces epithelial-mesenchymal transition via Arf6-ERK signaling in gastric cancer cells. Oncotarget 6: 7244-7261, 2015.

21. Singh A and Settleman J: EMT, cancer stem cells and drug resistance: An emerging axis of evil in the war on cancer. Oncogene 29: 4741-4751, 2010.

22. Mitra A, Mishra L and Li S: EMT, CTCs and CSCs in tumor relapse and drug-resistance. Oncotarget 6: 10697-10711, 2015.

23. Clark K, Karsch-Mizrachi I, Lipman DJ, Ostell J and Sayers EW: GenBank. Nucleic Acids Res 44: D67-D72, 2016.

24. Hu Z, Du J, Yang L, Zhu Y, Yang Y, Zheng D, Someya A, Gu L and $\mathrm{Lu} \mathrm{X}$ : GEP100/Arf6 is required for epidermal growth factor-induced ERK/Racl signaling and cell migration in human hepatoma HepG2 cells. PLoS One 7: e38777, 2012.

25. Hu Z, Xu R, Liu J, Zhang Y, Du J, Li W, Zhang W, Li Y, Zhu Y and Gu L: GEP100 regulates epidermal growth factor-induced MDA-MB-231 breast cancer cell invasion through the activation of Arf6/ERK/uPAR signaling pathway. Exp Cell Res 319: 1932-1941, 2013.

26. Davies JC, Tamaddon-Jahromi S, Jannoo R and Kanamarlapudi V: Cytohesin 2/ARF6 regulates preadipocyte migration through the activation of ERK1/2. Biochem Pharmacol 92: 651-660, 2014.

27. Akter H, Park M, Kwon OS, Song EJ, Park WS and Kang MJ: Activation of matrix metalloproteinase-9 (MMP-9) by neurotensin promotes cell invasion and migration through ERK pathway in gastric cancer. Tumour Biol 36: 6053-6062, 2015.

28. Li P, Jia YF, Ma XL, Zheng Y, Kong Y, Zhang Y, Zong S, Chen ZT and Wang YS: DEC2 suppresses tumor proliferation and metastasis by regulating ERK/NF- $\mathrm{BB}$ pathway in gastric cancer. Am J Cancer Res 6: 1741-1757, 2016.

29. Teng H, Huang Q and Chen L: Inhibition of cell proliferation and triggering of apoptosis by agrimonolide through MAP kinase (ERK and p38) pathways in human gastric cancer AGS cells. Food Funct 7: 4605-4613, 2016.

30. Eva R, Crisp S, Marland JR, Norman JC, Kanamarlapudi V, ffrench-Constant $\mathrm{C}$ and Fawcett JW: ARF6 directs axon transport and traffic of integrins and regulates axon growth in adult DRG neurons. J Neurosci 32: 10352-10364, 2012.

31. Torii T, Miyamoto Y, Yamamoto M, Ohbuchi K, Tsumura H, Kawahara K, Tanoue A, Sakagami H and Yamauchi J: Arf6 mediates Schwann cell differentiation and myelination. Biochem Biophys Res Commun 465: 450-457, 2015.

32. George AA, Hayden S, Stanton GR and Brockerhoff SE: Arf6 and the 5'phosphatase of Synaptojanin 1 regulate autophagy in cone photoreceptors. Inside Cell 1: 117-133, 2016.

33. Grossmann AH, Zhao H, Jenkins N, Zhu W, Richards JR, Yoo JH, Winter JM, Rich B, Mleynek TM, Li DY and Odelberg SJ: The small GTPase ARF6 regulates protein trafficking to control cellular function during development and in disease. Small GTPases: 1-12, 2016. doi: 10.1080/21541248.2016.1259710.

34. Chen PW, Jian X, Yoon HY and Randazzo PA: ARAP2 signals through Arf6 and Rac1 to control focal adhesion morphology. J Biol Chem 288: 5849-5860, 2013.

35. Hongu T, Funakoshi Y, Fukuhara S, Suzuki T, Sakimoto S, Takakura N, Ema M, Takahashi S, Itoh S, Kato M, et al: Arf6 regulates tumour angiogenesis and growth through HGF-induced endothelial $\beta 1$ integrin recycling. Nat Commun 6: 7925, 2015.

36. Bourmoum M, Charles R and Claing A: The GTPase ARF6 controls ROS production to mediate angiotensin II-induced vascular smooth muscle cell proliferation. PLoS One 11: e0148097, 2016.

37. Sabe H, Hashimoto S, Morishige M, Ogawa E, Hashimoto A, Nam JM, Miura K, Yano $H$ and Onodera Y: The EGFR-GEP100-Arf6-AMAP1 signaling pathway specific to breast cancer invasion and metastasis. Traffic 10: 982-993, 2009.

This work is licensed under a Creative Commons Attribution-NonCommercial-NoDerivatives 4.0 International (CC BY-NC-ND 4.0) License. 